\title{
Endometriosis: Diagnosis and Treatment: From Fingernails to the Robot
}

Elizabeth A.. Buescher, Camran Nezhat

1.

Corresponding author: Elizabeth A.. Buescher, lizziebuescher@hotmail.com

\begin{abstract}
Endometriosis, the presence of endometrial glands and stroma outside of the endometrial cavity, is a disease that causes pelvic pain and infertility. Unfortunately, this disease is often undiagnosed for years, resulting in a delay in treatment. Therefore, the clinician must maintain a high index of suspicion to minimize the morbidity of the disease. In recent years, there have been many advances in the treatment of endometriosis, with video-assisted laparoscopic surgery (VALS) at the forefront of these changes. Because endometriosis can affect many organ systems, an interdisciplinary approach is often needed to treat these patients. This review will address diagnosis, work-up, and management of this complex disease.
\end{abstract}

Categories: Obstetrics/Gynecology, General Surgery

Keywords: laparoscopy, extra-genital, pathogenesis, robotics, chronic pelvic pain, endometriosis

\section{Introduction And Background}

The classic triad of endometriosis is dysparunea, dysmenorrhea, and infertility. However, this triad often oversimplifies a complex disease that has been causing chronic pain and infertility for millennia. Pathologically, endometriosis is characterized by the presence of endometrial cells outside of the endometrial cavity. Most commonly, it is located on the peritoneal surface or other pelvic organs, but endometriosis has been found in every organ of the body except for in the spleen [1-2]. The endometrial tissue is activated by estrogen, which can cause the endometrial tissue to spread and cause pain for the patient [3].

\section{Review}

\section{Pathogenesis of Endometriosis}

The pathogenesis of endometriosis remains unclear. Sampson is generally credited with the most popular theory in 1927 [4-5]. However, retrograde menstruation was actually suggested in the 1690's by both Schroen and Ruysch [6]. They suggested that retrograde menstruation caused the menstrual blood to spill out of the fallopian tubes and into the pelvic cavity, causing implants of endometriosis. Another theory is the coelomic metaplasia theory, which suggests that peritoneal tissue undergoes metaplastic change to endometrial tissue before endometriosis can implant. This theory is supported by the fact that both endometrial tissue and peritoneal tissue have a common precursor: coelomic tissue $[5,7]$. There is some thought that stem cells may play a role through this mechanism. The third theory is the theory of lymphatic or hematologic spread.

This theory helps explain the presence of endometriosis in areas remote from the pelvis [5, 8-9]. The final theory is that of direct transplantation. This explains the presence of endometriosis in surgical scars. In vivo, this is referred to as autotransplantation [5, 10-15]. We believe that the hematologous/lymphatic spread theory likely exacerbates the disease, but is not the entity that initially starts the disease. Retrograde menstruation, metaplastic change, and direct transplantation likely play a role as well, but may not be the inciting event that starts the chain reaction of endometriosis.

\section{Prevalence}

The prevalence of the disease is uncertain as this disease is under diagnosed and patients are often misdiagnosed for years before they are correctly diagnosed with endometriosis. Most clinicians consider endometriosis to have a prevalence of $6-10 \%$ prevalence in the general population, with that prevalence rising to 35-50\% in women with pain and/or infertility [3]. A diagnosis of endometriosis should also be considered in unexplained infertility. In our practice, which specializes in endometriosis, infertility, and chronic pelvic pain, we have noted a prevalence of over $90 \%$ of pathology-proven endometriosis.

It is important to note that the clinician must have a high index of suspicion to diagnose endometriosis.

\section{Patient evaluation}

When a patient presents with pelvic pain, the clinician must first work to determine which organ systems are involved. During the history, the patient should be asked: 
1. When did the pain start?

2. Is the pain associated with ovulation or menses?

3. Does the patient have dysparunea?

4. Are there any GI symtoms: nausea, vomiting, diarrhea, constipation?

5. Are there any GU symptoms: dysuria, urgency, frequency?

6. Has the patient ever been sexually abused?

7. How long is the patient's menstrual cycle?

8. How heavy is the patient's menstrual cycle?

9. Does the patient have unexplained infertility?

These questions are important, but one should also be aware that endometriosis can cause pain that is not associated with menstrual cycle. In addition, a large number of patients with "unexplained infertility" who do not have dysmenorrhea are found to have endometriosis at laparoscopy. Again, one must maintain a high index of suspicion.

Once the history has been taken, the patient should undergo a complete physical exam, including a pelvic exam and a speculum exam. The best time to examine a patient is during the luteal phase or at the time of her menses because the endometriotic lesions are enlarged and more easily palpable. During the pelvic exam, the provider should feel for the presence of nodularity on the uterosacral ligaments, which is consistent with endometriosis. The physician should also see if the uterus is fixed or mobile, and assess for adnexal fullness, cervical motion tenderness, and the presence of levator ani spasm.

After the exam, the patient should have a transvaginal ultrasound to look for the presence of ovarian cysts. If an ovarian cyst has a ground glass appearance due to low level homogenous echoes on ultrasound, that is concerning for an endometrioma [16]. However, it is important to note that sonographic findings alone cannot rule out malignancy.

The ultrasound should include a color Doppler ultrasound to look at the blood flow to the uterus, tubes, and ovaries. The presence of hypervascularity in the uterus is suggestive of adenomyosis, which is essentially endometriosis of the uterus. Other signs of adenomyosis include dimensions that show a globular uterus, non-symmetric anterior-posterior uterine measurements, a heterogenous myometrium, and the presence of tiny endometrial cysts in the myometrium [16].

Depending on the results of the above evaluation, lab tests may or may not be indicated. If a patient has GI symptoms, consider stool cultures, colonoscopy, and a complete metabolic panel. If a patient has GU symptoms, consider a urinalysis, a urine culture, and a cystoscopy. If the patient has a history of sexual abuse, unprotected intercourse, or abnormal vaginal discharge, consider cervical cultures.

\section{Treatment of endometriosis}

Depending on the severity of symptoms and the patient's desires, one can proceed with either medical or surgical treatment. In our practice, when patients present with severe pain refractory to medical management or infertility in spite of treatment with assisted reproduction techniques, we proceed with surgical treatment and then use medical treatment to keep the endometriosis at bay, provided the patient does not desire immediate fertility. For young infertile patients with endometriosis, we recommend natural conception after surgery provided the partner has a normal sperm count. For older patients, IVF may be necessary for fertility [17].

\section{Video-assisted laparoscopic treatment of endometriosis}

Like the pelvic exam, video-assisted laparoscopy is also best performed during the luteal phase so that the lesions are larger and more easily seen. Laparoscopic treatment of extensive endometriosis including endometriosis of the bladder, bowel, and ureter was first reported in the 1980's [18-20]. Laparoscopic diagnosis and eradication of all forms of endometriosis are effective and may now be considered the gold standard of clinical care for women with endometriosis-related pain and infertility. The recognized advantages of video-assisted laparoscopy include faster patient recovery, smaller scars, lesions adhesions, better results, and reduced cost [21].

Historically, excision of endometriosis dates back to a time when surgeons used their fingernails to excise endometriosis [6]. Modern surgical tools have expanded the gynecologic surgeons ability to treat endometriosis. These include the $\mathrm{CO} 2$ laser, scissors with monopolar electrocautery, bipolar electrocautery, Harmonic Ace ultrasonic energy (Ethicon, Somerville, NJ), the PlasmaJet (Plasma Surgical, Inc., Roswell, GA), and the da Vinci robot (Intuitive Surgical, Inc, Sunnyvale, CA) all of which allow resection, cauterization, or vaporization of endometriosis. In a recent prospective study, laparoscopic excision of endometriosis significantly reduced pain and improved quality of life for up to five years [22]. There may be a continuing role for hysterectomy in the management of endometriosis, but the evidence for concomitant oophorectomy is less convincing. In general, conservative surgery should be considered first. 


\section{Cureus}

initially had to prove that laparoscopy was equivalent, if not superior to laparotomy. It is a matter of academic interest to understand the history of laparoscopy and how it relates to the surgical treatment of endometriosis, and see the rejection that laparoscopists had to overcome to gain acceptance [23-24]. The results of endoscopy and laparotomy are judged by many factors [25]. Studies comparing adhesion formation after laparotomy versus laparoscopy have repeated shown laparoscopy to have less adhesion formation [2635]. Results from general surgery studies have also supported the fact that laparoscopy causes less adhesions than laparotomy. In 2001, Polymeneas compared adhesion formation in laparoscopic cholecystectomy versus open cholecystectomy. The investigators found that $100 \%$ of patients with a prior open cholecystectomy had dense adhesion formation, whereas $45 \%$ of those patients who underwent laparoscopic cholecystectomy had loose or minimal adhesions at repeat surgery [36].

Once it was established that laparoscopy was superior to laparotomy, it was compared to vaginal surgery. A 2005 study from Nascimeno comparing morphine requirements in patients with vaginal hysterectomy versus laparoscopic hysterectomy found that those patient who underwent laparoscopic hysterectomy required less morphine and were on NSAIDs for fewer days following surgery [37].

\section{Appearance of Endometriosis}

Video-assisted laparoscopic assessment in combination with histologic examination of the treated lesions remains the gold standard for diagnosis of endometriosis. Knowledge of the most common locations of endometriosis is required for accurate visual inspection of the pelvic and abdominal cavities. Several different forms of endometriosis must be considered during laparoscopic visualization: peritoneal implants, endometriomas, deep infiltrating lesions of the rectovaginal septum, and extragenital endometriosis. An increased awareness of the variations in the appearance of endometriotic lesions has resulted in an almost two-fold increase in the diagnosis of endometriosis at laparoscopy [38].

Peritoneal implants are most commonly localized to the uterosacral ligaments, cul-de-sac, ovarian fossa, and adjacent pelvic sidewalls (Figure 1). Less frequently, implants may also be found in the upper abdomen as well as on the surface of the ovary, bladder and the bowel (predominantly rectum, sigmoid colon, appendix, and cecum (Figure 2).

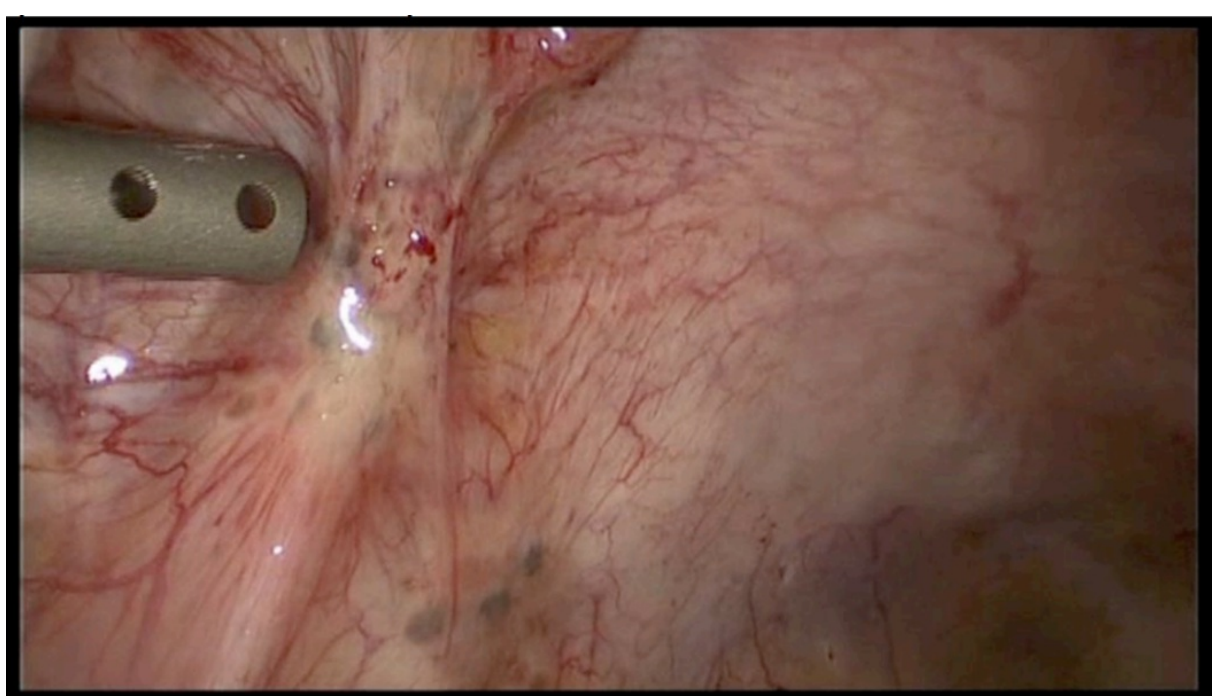

FIGURE 1: Dark “powder burn” lesions

Endometriosis is commonly seen at video-assisted laparoscopy as the dark "powder burn" lesions on the pelvic sidewall as shown here 


\section{Cureus}

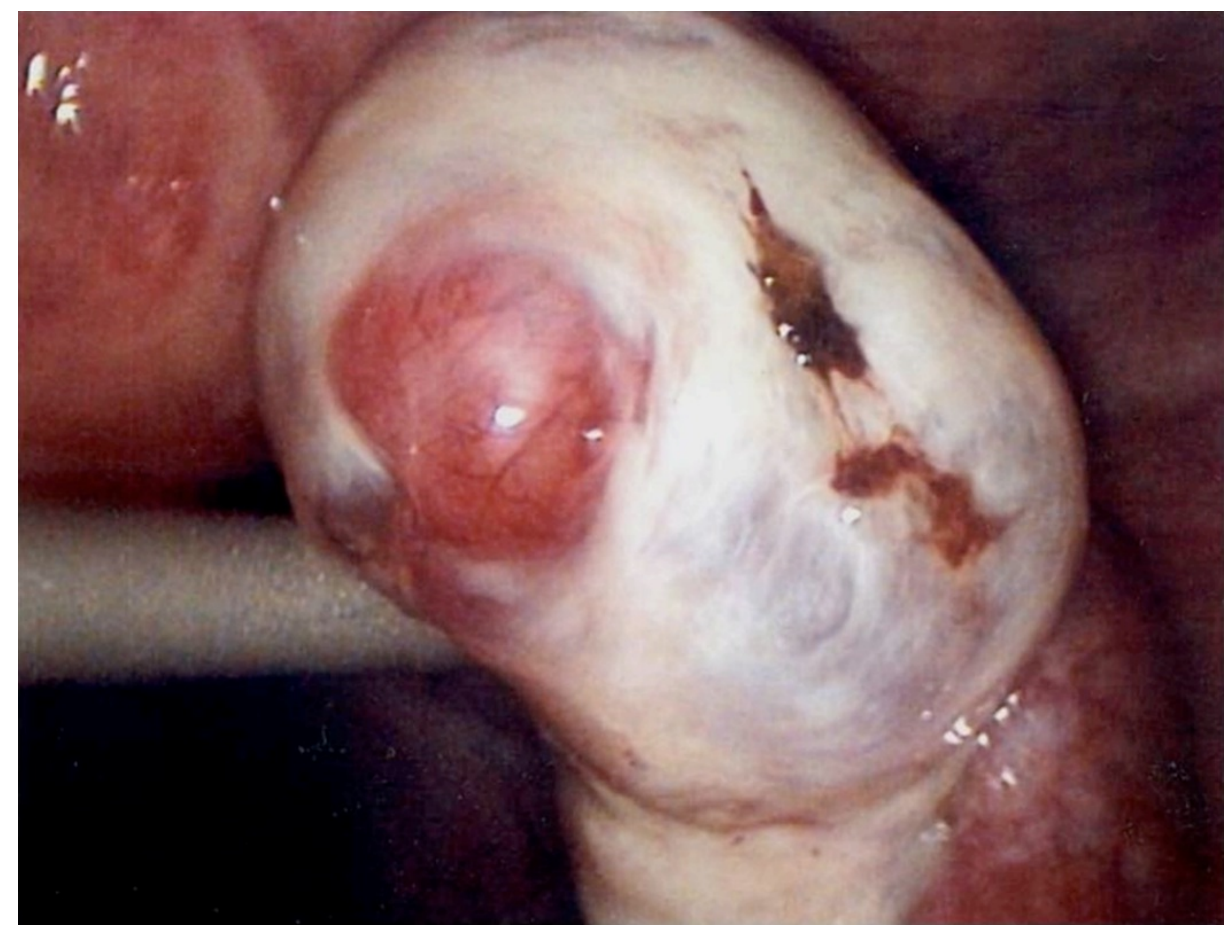

FIGURE 2: Dark lesion on ovary

Endometriosis can be seen as the dark lesion on this ovary during video-assisted laparoscopy.

Hence, careful and close inspection of the entire peritoneal cavity should be performed. In addition, the camera should be turned towards the diaphragm and liver to look for signs of endometriosis there.

Magnification obtained during laparoscopy depends on the distance between the laparoscope and the area inspected, as the closer the laparoscope is to the tissue, the greater the magnification.

\section{Endometriomas}

Endometriomas, commonly referred to as "chocolate cysts," are one of the most common manifestations of endometriosis (Figure 3).

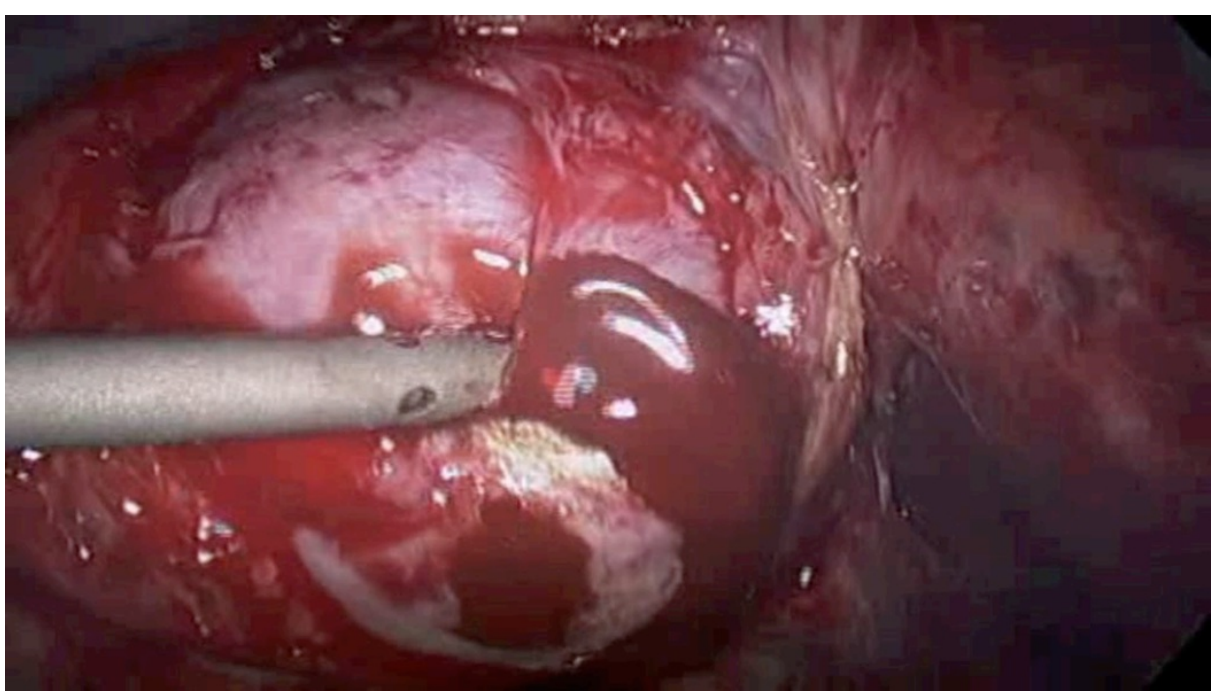

FIGURE 3: Endometrioma immediately prior to resection. The suctionirrigator can be used to drain the endometrioma without spilling its contents. 
Nezhat, et al. [39-40], classified endometriomas as Types I or Type II. Type I endometriomas are primary endometriomas. They are small, usually less than $5 \mathrm{~cm}$ in size, and contain dark fluid. They develop from surface endometriotic implants and are difficult to excise surgically. Microscopically, endometrial glands and stroma are always present. These endometriomas are thin endometrial glands and stroma, which adhere to the surface of the ovary and invaginate into the ovary and are difficult to remove. Their video-assisted laparoscopic removal requires an expert surgeon. In less experienced hands, the ovarian stroma could be compromised. Type I endometriomas can be treated by either vaporization or a combination of vaporization and excision. If the surgeon is unable to remove the cyst wall without compromising ovarian stroma, then the endometrioma should be aspirated, biopsies should be taken, and the cyst wall should be vaporized. This technique has recently become more popular as it is surgically simpler and less time consuming

than excising the cyst wall in its entirety [41]. Type II endometriomas are primarily functional cysts that have been invaded by endometriosis [39-40]. Type II endometriomas are much easier to remove than Type II endometriomas. Regardless of the type of endometrioma encountered at surgery, they will not resolve spontaneously and should be surgically excised and the cyst wall sent for pathologic evaluation.

\section{Computer-enhanced technology: the robot}

With the introduction of the da Vinci Surgical System (Intuitive Surgical, Inc. Sunnyvale, CA), commonly referred as "the robot" in 1999, surgical treatment of endometriosis has become more accessible for both patients and physicians. As physicians and hospitals work to integrate the robot into their patient care, most have noted that having a dedicated team for the robot greatly reduces operating time as docking the robot can be time consuming for those who are not proficient at it. Studies have shown that robotic surgeries take longer to complete than their laparoscopic counterparts, but the operating time does decrease as more cases are performed [42]. The da Vinci robot has articulating instruments that more closely resemble the movements that the human wrist can perform at laparotomy as opposed to the movements that can be done by laparoscopy (Figure 4 ).

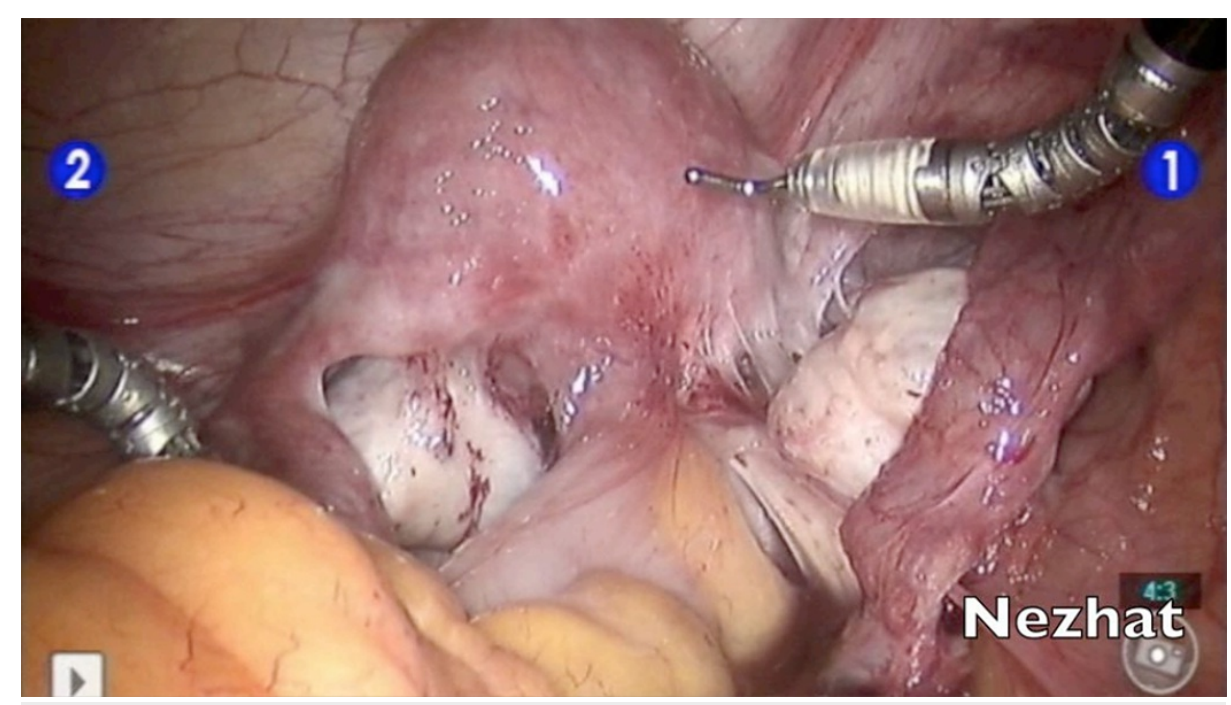

\section{FIGURE 4: The da Vinci robot is used to surgically treat severe endometriosis}

The da Vinci robot is used to surgically treat severe endometriosis

This articulation makes suturing and other surgical procedures easier with the robot than with traditional laparoscopy. The primary advantage of the robot over laparoscopy is that the robot is easier to master. Therefore, it makes minimally invasive surgery easier for the physician, meaning more physicians are able to provide their patients with a minimally invasive surgical treatment.

\section{Medical management of endometriosis}

Because endometriosis is hormonally responsive, postoperatively it is important to regulate the patient's hormone levels. We generally start the patients who do not desire immediate fertility on continous oral contraceptive pills. Studies show that both continuous use and cyclic use have beneficial results, and the decision to use one or the other should be made on a patient-by-patient basis [43].

GnRH agonists can also be used in the short-term for patients with chronic pelvic pain to induce a medical menopause since endometriosis is estrogen-dependent. The combination of anastrozole plus goserelin can be used to reduce pain and reduce recurrences [44-45]. Similarly, letrozole has been reported to decrease 


\section{Cureus}

pelvic pain and decrease dysparunea $[44,46]$.

In addition, pelvic floor physical therapy and acupuncture are helpful to treat the muscle spasms of chronic pain. Pelvic floor physical therapy requires dedication and patience on the part of the physician and the patient, as it often takes two to three months of treatment for the patient to achieve the full benefit.

\section{Extra-genital endometriosis}

Although endometriosis is generally limited to the pelvic peritoneum and pelvic organs, endometriosis can also be found throughout the body. Endometriosis may spread to the urinary system in $1 \%$ to $2 \%$ of women with symptomatic endometriosis. Endometriosis of the urinary tract tends to be superficial but may be invasive and cause complete ureteral obstruction [47]. Clinicians should consider endometriosis in cases of refractory and unexplained urinary complaints. If urinary tract endometriosis is suspected, an intravenous pyelogram (IVP) or CT with IV contrast and delayed images to evaluate the ureters, ultrasound of the kidneys, and a routine blood and urine work-up may be indicated. In selected cases of recurrent hematuria, cystoscopy is suggested (Figure 5). When bladder or ureteral endometriosis is suspected, urologic consult may be helpful.

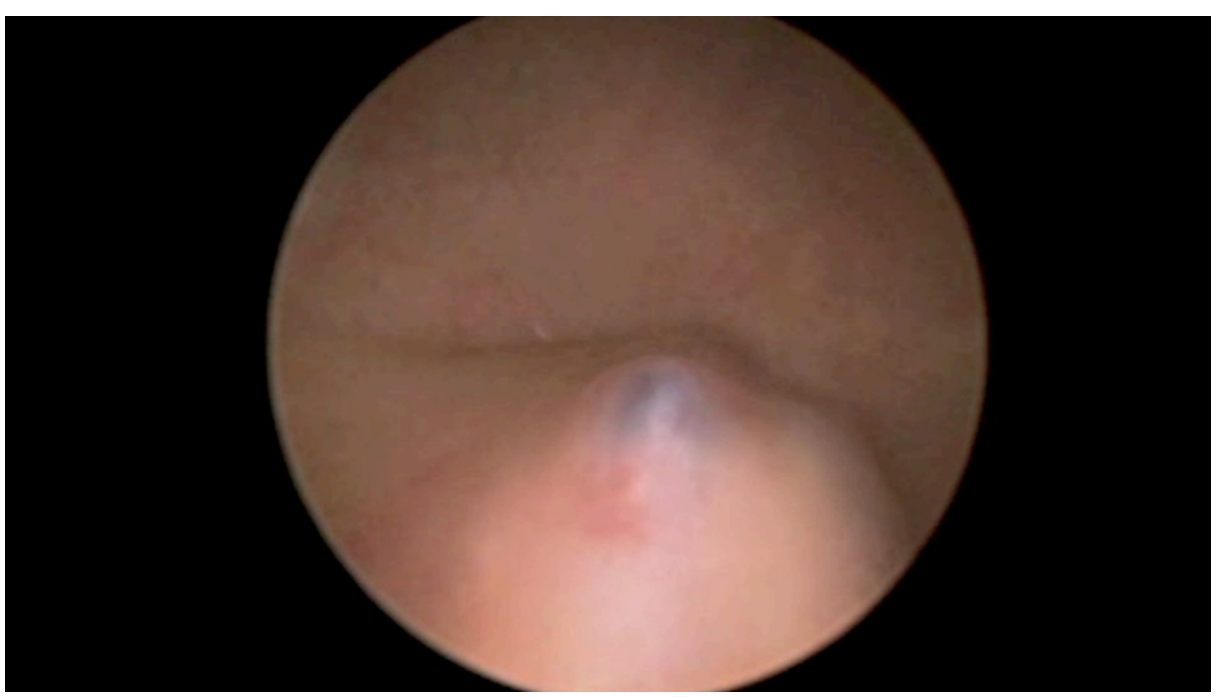

FIGURE 5: A lesion of bladder endometriosis

A lesion of bladder endometriosis is seen at cystoscopy.

Gastrointestinal endometriosis was described in 1901 by Cullen [6] and in 1922 by Sampson [48] during the histologic examination of resected sigmoid colon that had been diagnosed intraoperatively as a carcinoma. The gastrointestinal tract is believed to be involved in $3 \%$ to $37 \%$ of women with endometriosis [4950]. However, in a specialized practice, the number of patients with bowel involvement may be as high as $50 \%$ if patients with serosal and subserosal lesions are included. Endometriotic implants may be found between the small intestine and the anal canal. Intestinal endometriosis involves the rectum and sigmoid colon in $76 \%$ of cases (Figure 6 ), the appendix in $18 \%$ (Figure 7 ), and the cecum in $5 \%$. The clinical presentation varies from an incidental finding at laparoscopy or laparotomy to bowel obstruction [51]. Operative laparoscopy is performed to treat endometriotic implants on the intestinal wall, appendix, and rectovaginal space [20]. The surgery performed varies depending on the patient but can include appendectomy [52], disk excision, or bowel resection. Bowel resection should be reserved for those patients who continue to have symptoms despite more conservative forms of treatment [20]. 


\section{Cureus}

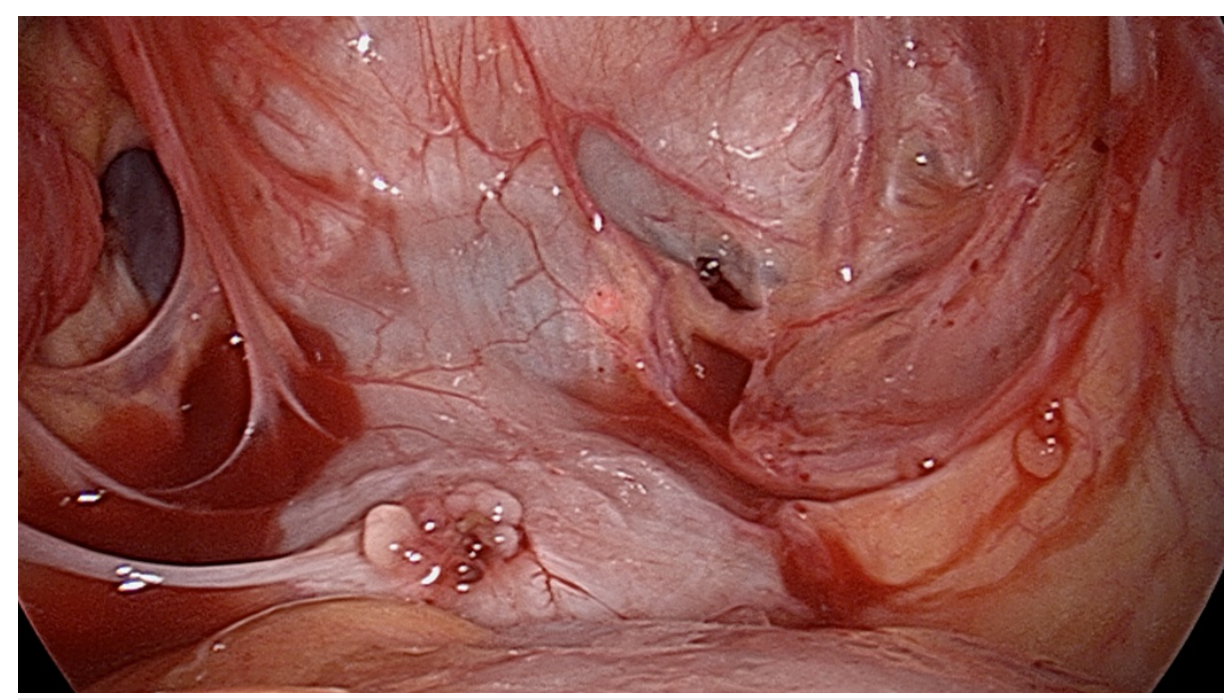

FIGURE 6: Endometriosis

The bubbles on the rectum of this patient are endometriosis.

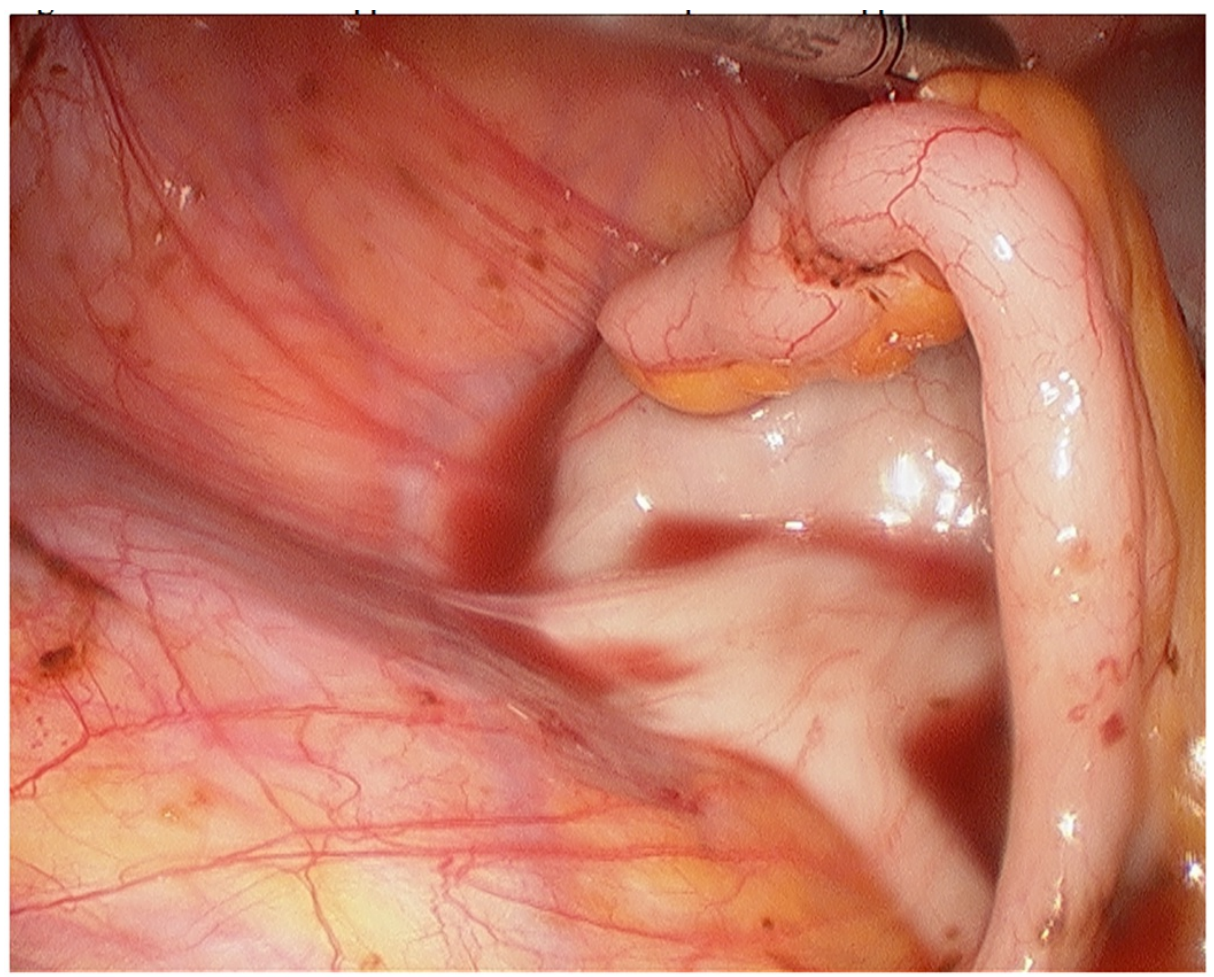

FIGURE 7: Endometriosis

Endometriosis appears as black and red spots on this appendix.

The diaphragm is rarely a reported site of endometriosis [53]. Women should be asked about pleuritic, shoulder, or upper abdominal pain occurring with menses because they do not make the connection between these distant anatomic landmarks. The laparoscope is excellent for diagnosing and possibly treating endometriosis on the diaphragm, which is difficult to reach by laparotomy [2]. Endometriosis of the liver is even more rare than diaphragmatic endometriosis, but can cause cyclic right upper quadrant pain [54].

In patients who present with catamenial pneumothorax, catamenial hemoptysis, catamenial hemothorax, or lung nodules, thoracic endometriosis must be considered. Catamenial pneumothorax is the most common presenation of thoracic endometriosis [55]. 


\section{Cureus}

If it has been determined preoperatively that the patient may have thoracic endometriosis, video-assisted thoracic surgery should be performed by a cardiothoracic surgeon at the time of laparoscopy. During the VATS procedure, any endometriotic implants should be ablated or resected (Figure 8). In addition, any scarring of the lung to the thoracic sidewall should be treated surgically [2].

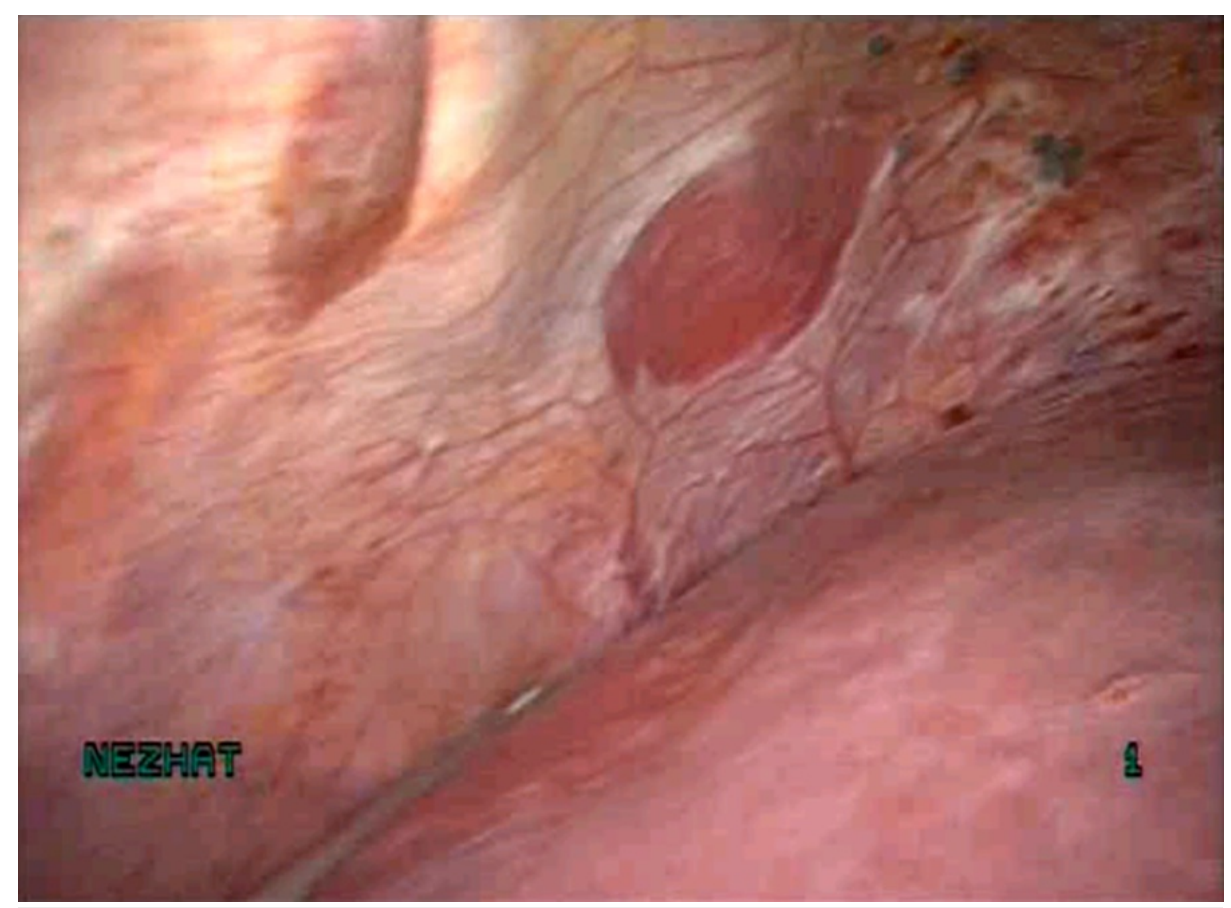

FIGURE 8: Endometriosis

During a VATS procedure, endometriosis is seen on the thoracic sidewall.

\section{Future directions}

As for the "etiologic chaos" that persists, drawing from over 30 years of endometriosis research and surgical experience, which includes by now approximately 14,000 surgeries, several important insights come to mind. The first is that we believe it's time to radically reevaluate how endometriosis's four main theories of pathogenesis are conceptualized. In our view, all four theories are partially correct. We arrived at this hybrid theory after observing what we suspect to have been all four pathogeneses at play: 1) retrograde menstrual endometrium implanting on peritoneal surfaces and transforming into pathology; 2) a pattern of coelomic metaplastic differentiation of mesothelial cells into endometrium-like tissue; 3) lymphatic and venous spread transporting and depositing endometriosis into areas which cannot be explained by the other theories; and 4) iatrogenic or direct transplantation, which would explain the presence of endometriosis in surgical scars. Rather than viewing these theories from a zero-sum standpoint, we believe that by conceptualizing endometriosis through the framework of an integrated model, this could potentially lead to significant improvements in preventive and treatment strategies, as well as potentially lead us more closely toward a cure.

There is escape of endometrial glands and stroma from the fallopian tubes at the time of diagnostic laparoscopy while hysteroscopy is being performed simultaneously (Figures 9-10). 


\section{Cureus}

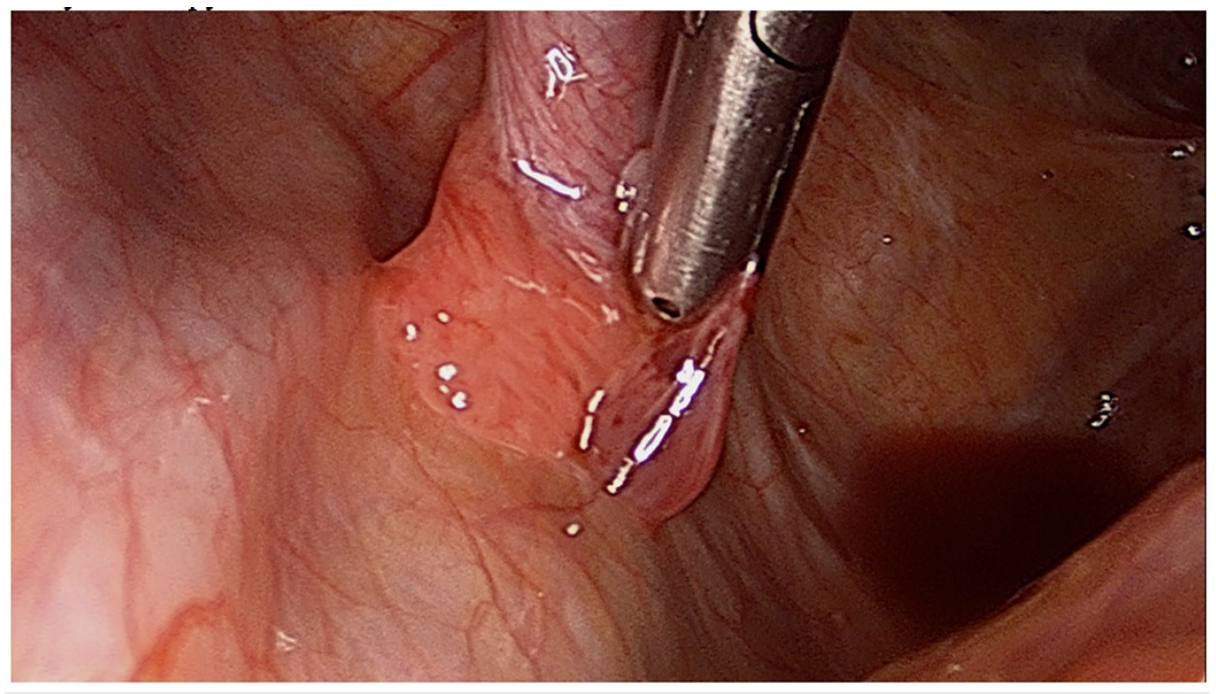

FIGURE 9: Endometrial glands and stroma escaping from the fallopian tube

Endometrial glands and stroma escaping from the fallopian tube at the the time of hysteroscopy

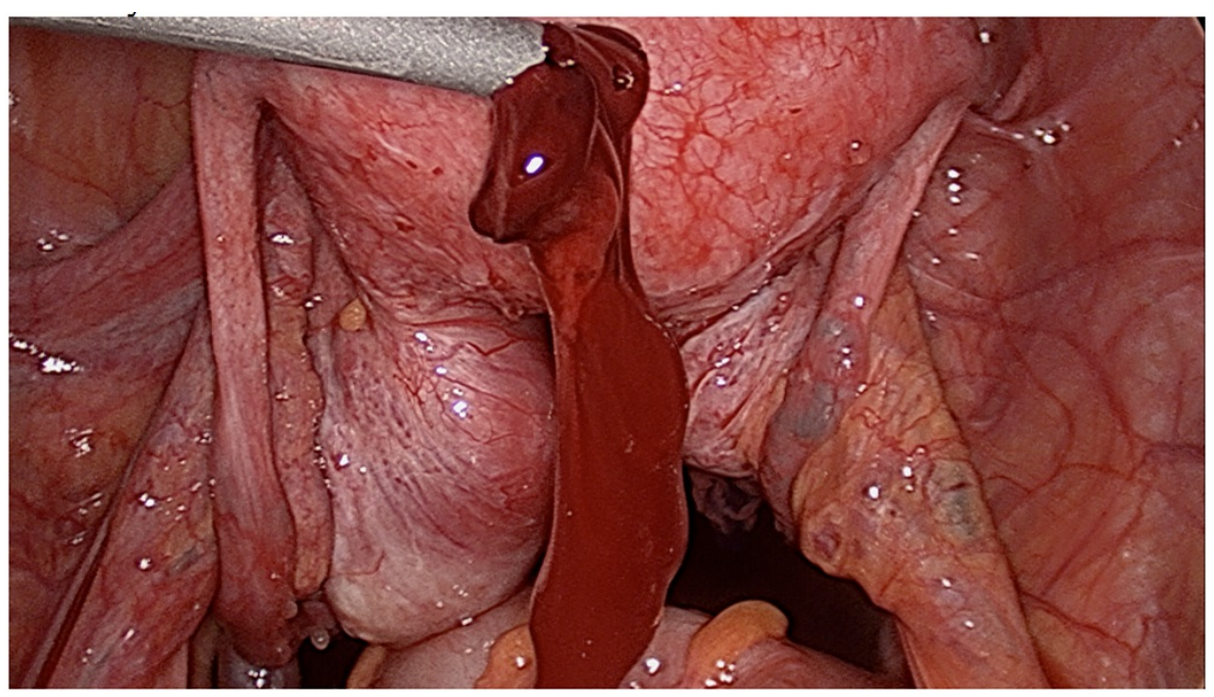

\section{FIGURE 10: Endometrial glands}

Immediately after hysteroscopy, these blebs of endometrial glands and stroma are easily removed from the pelvis and sent for pathologic evaluation. The pathology is invariably endometriosis.

On countless occasions over more than three decades, we have observed this phenomenon repeatedly. Pathologic diagnosis of endometriosis has been noted $100 \%$ of the time. Additionally, we have observed different stages of progression of these lesions attaching themselves to different organs of the pelvis (Figure 11). Thus, we have no doubt that retrograde menstruation is one of the causes of endometriosis. 


\section{Cureus}

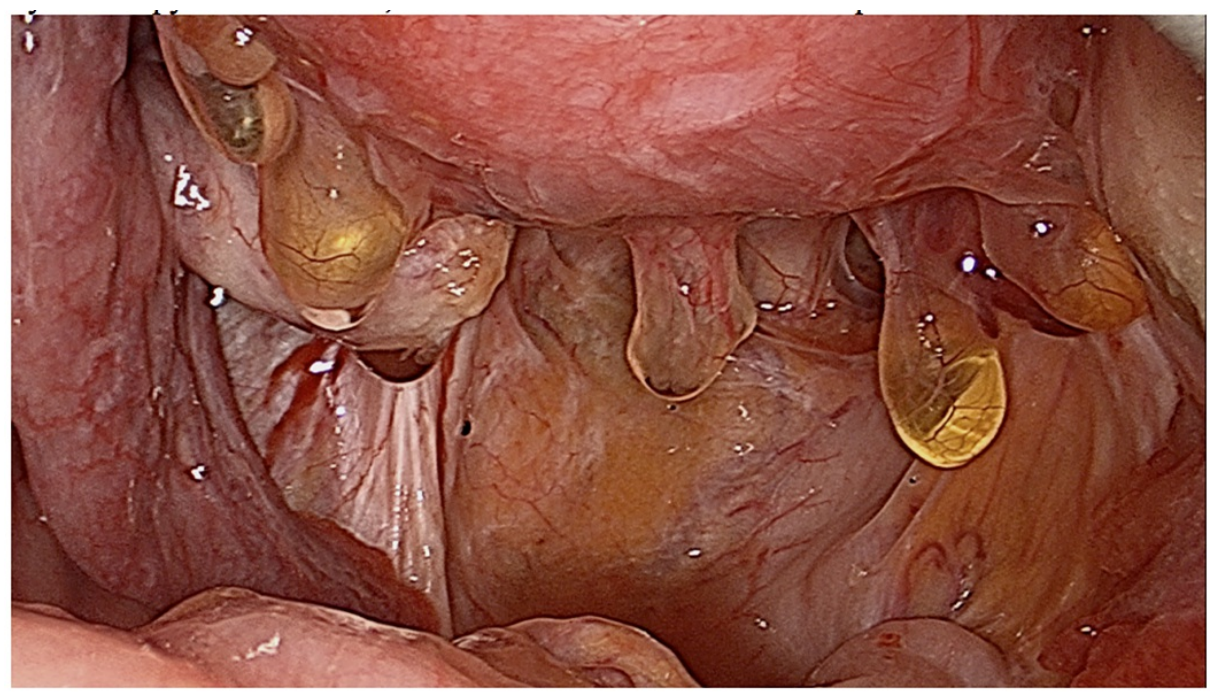

\section{FIGURE 11: Endometrial glands and stroma}

Endometrial glands and stroma have attached to the posterior uterus after hysteroscopy. If left behind, this can lead to obliteration of the posterior cul de sac.

With regard to the assumption that resection of endometriosis can be cured, we have observed over and over new implants of endometriosis exactly at the center of the craters or at the periphery of previously resected disease (Figure 12).

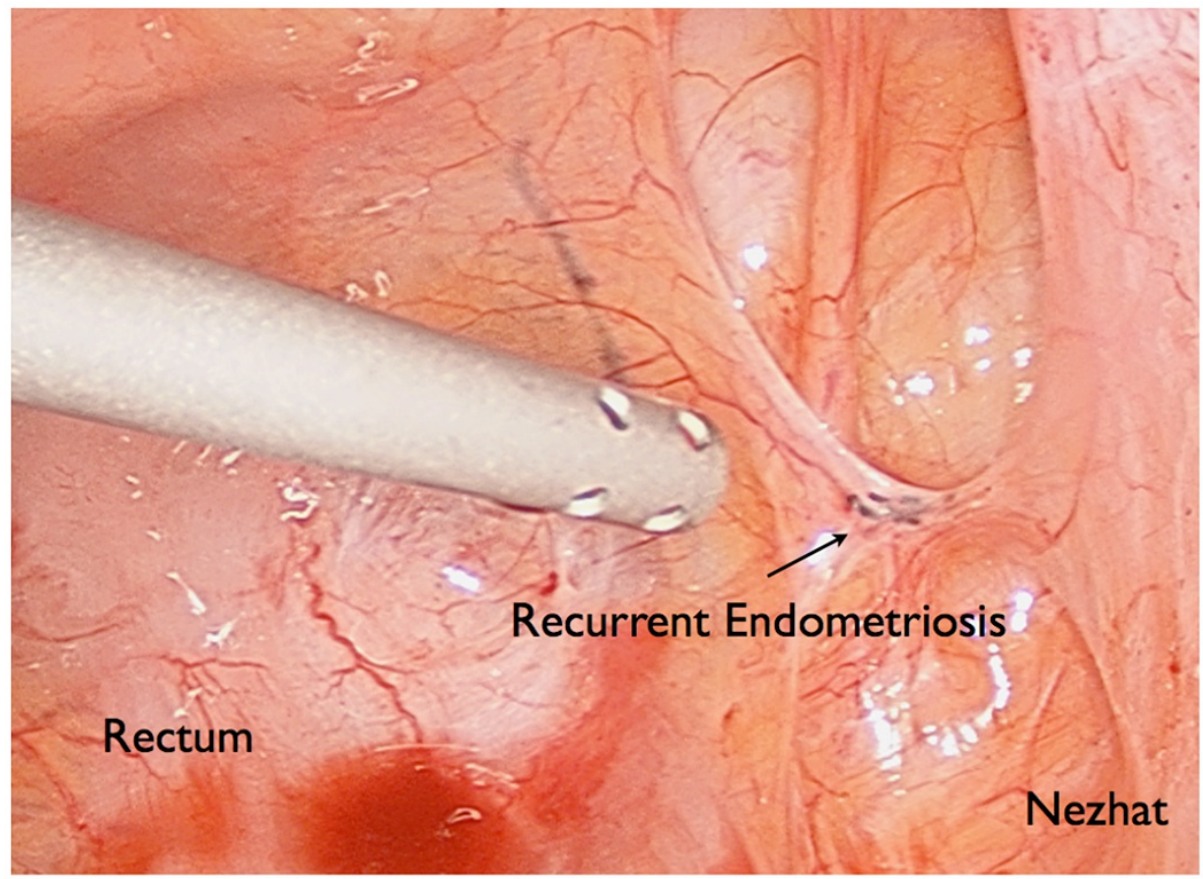

\section{FIGURE 12: Endometriosis}

Endometriosis can recur even at sites of previous excision. This image clearly shows the recurrence of endometriosis at the left pararectal site of previous excision.

Additionally, on rare occasions over the past several decades we have noticed severe dissemination of endometriosis on repeat laparoscopy on patients with mild to moderate endometriosis at the initial procedure within the three years. 


\section{Conclusions}

As providers of women's health, we must always strive to provide the highest level of care for our patients. Women with chronic pelvic pain or infertility often present a challenge to the clinician as they often present with multiple previous diagnoses and may be frustrated with the medical system. When a patient in her reproductive years presents with pelvic pain or infertility, endometriosis should be considered as a diagnosis and treatment started. Endometriosis treatment often requires a multidisciplinary approach: pain management to help manage chronic pain, physical therapy, and those patients with extragenital endometriosis may need co-management with urology, colorectal surgery, gastroenterology, and cardiovascular surgery. However, with appropriate care, the majority of these patients can be relieved of their symptoms and achieve fertility.

\section{Additional Information \\ Disclosures}

Conflicts of interest: In compliance with the ICMJE uniform disclosure form, all authors declare the following: Payment/services info: All authors have declared that no financial support was received from any organization for the submitted work. Financial relationships: All authors have declared that they have no financial relationships at present or within the previous three years with any organizations that might have an interest in the submitted work. Other relationships: All authors have declared that there are no other relationships or activities that could appear to have influenced the submitted work.

\section{References}

1. Veeraswamy A, Lewis M, Mann A, Kotikela S, Hajhosseini B, Nezhat C: Extragenital endometriosis. Clin Obstet Gynecol. 2010, 53:449-66.

2. Nezhat C, Nicoll LM, Bhagan L, et al: Endometriosis of the diaphragm: four cases treated with a combination of laparoscopy and thoracoscopy. J Minim Invasive Gynecol. 2009, 16:573-80. 10.1016/j.jmig.2009.06.012

3. Giudice LC, Kao LC: Endometriosis. Lancet. 2004, 364:1789-99.

4. Sampson JA: Peritoneal endometriosis due to menstrual dissemination of endometrial tissue into the peritoneal cavity. Am J Obstet Gynecol. 1927, 14:422-69.

5. Jensen JR, Coddington CC 3rd.: Evolving spectrum: the pathogenesis of endometriosis . Clin Obstet Gynecol. 2010, 53:379-88. 10.1097/GRF.0b013e3181db7b84

6. Nezhat C, Nezhat F, Nezhat C: Endometriosis: ancient disease, ancient treatments. . Fertil Steril. 2012, 98:162. 10.1016/j.fertnstert.2012.08.001

7. Ferguson BR, Bennington JL, Haber SL: Histochemistry of mucosubstances and histology of mixed mullerian pelvic lymph node glandular inclusions. Evidence for histogenesis by mullerian metaplasia of coelomic epithelium. Obstet Gynecol. 1969, 33:617-625.

8. Halban J: Hysteroadenosis metastica (die lymphogene genes dersog. adenofibromatosis hetertopica). Wein Klin Wochenschr. 1924, 37:1205.

9. Sampson JA: Metastatic or Embolic Endometriosis, due to the Menstrual Dissemination of Endometrial Tissue into the Venous Circulation. Am J Pathol. 1927, 3:93-110.

10. Bulun SE: Endometriosis. N Engl J Med. 2009, 360:268-79. 10.1056/NEJMra0804690

11. Hornung D, Ryan IP, Chao VA, Vigne JL, Schriock ED, Taylor RN: Immunolocalization and regulation of the chemokine RANTES in human endometrial and endometriosis tissues and cells. J Clin Endocrinol Metab. 1997, 82:1621-1628.

12. Kao LC, Germeyer A, Tulac S, et al: Expression profiling of endometrium from women with endometriosis reveals candidate genes for disease--based implantation failure and infertility. Endocrinology. 2003, 144:2870-2881.

13. Osteen KG, Bruner KL, Sharpe--Timms KL: Steroid and growth factor regulation of matrix metalloproteinase expression and endometriosis. Semin Reprod Endocrinol. 1996, 14:247-255.

14. Tseng JF, Ryan IP, Milam TD, et al: Interleukin--6 secretion in vitro is up-- regulated in ectopic and eutopic endometrial stromal cells from women with endometriosis. J Clin Endocrinol Metab. 1996, 81:1118-1122.

15. Redwine DB: Was Sampson wrong?. Fertil Steril. 2002, 78:686-693.

16. Kuligowska E, Deeds L 3rd., Lu K 3rd.: Pelvic pain: overlooked and underdiagnosed gynecologic conditions. Radiographics. 2005, 25:3-20.

17. Littman E, Giudice L, Lathi R, Berker B, Milki A, Nezhat C: Role of laparoscopic treatment of endometriosis in patients with failed in vitro fertilization cycles. Fertil Steril. 2005, 84:1574-1578.

18. Nezhat C, Nezhat F: Operative laparoscopy (minimally invasive surgery): state of the art . J Gynecol Surg. 1992, 81:111-141.

19. Berker B, Hsu T, Lee K, Nezhat C, Nezhat FR, Nezhat C: Laparoscopic treatment of endometriosis. Nezhat's operative gynecologic laparoscopy and hysteroscopy. Nezhat C, Nezhat FR, Nezhat C (ed): Cambridge University Press, Cambridge ; New York; 2008. 263-303.

20. Nezhat C, Hajhosseini B, King LP: Laparoscopic management of bowel endometriosis: predictors of severe disease and recurrence. Jsls. 2011, 15:431-438. 10.4293/108680811X13176785203752

21. Luciano AA, Lowney J, Jacobs SL: treatment of endometriosis-- associated infertility. Therapeutic, economic and social benefits. J Reprod Med. 1992, 37:573-576.

22. Davis CJ, McMillan L: Pain in endometriosis: effectiveness of medical and surgical management . Curr Opin Obstet Gynecol. 2003, 15:507-512.

23. Page B: Camran Nezhat \& the Advent of Advanced Operative Video---Laparoscopy . Nezhat's History of Endoscopy---A Historical Analysis of Endoscopy's Ascension Since Antiquity. Nezhat C (ed): Endo Press, 
Tuttlingen, Germany; 2011.

24. Kelley WE Jr.: The evolution of laparoscopy and the revolution in surgery in the decade of the 1990s . Jsls. 2008, 12:351-357.

25. Olive DL, Martin DC: Treatment of endometriosis--associated infertility with CO2 laser laparoscopy: the use of one-- and two--parameter exponential models. Fertil Steril. 1987, 48:18-23.

26. Filmar S, Gomel V, McComb PF: Operative laparoscopy versus open abdominal surgery: a comparative study on postoperative adhesion formation in the rat model. Fertil Steril. 1987, 48:486-489.

27. Luciano AA, Maier DB, Koch EI, Nulsen JC, Whitman GF: A comparative study of postoperative adhesions following laser surgery by laparoscopy versus laparotomy in the rabbit model. Obstet Gynecol. 1989, 74:220224 .

28. Maier DB, Nulsen JC, Klock A, Luciano AA: Laser laparoscopy versus laparotomy in lysis of pelvic adhesions . J Reprod Med. 1992, 37:965-968.

29. Hackethal A, Sick C, Szalay G, et al: Intra--abdominal adhesion formation: does surgical approach matter? Questionnaire survey of South Asian surgeons and literature review. J Obstet Gynaecol Res. 2011, 37:13821390.

30. Diamond MP, Daniell JF, Feste J, et al: Adhesion reformation and de novo adhesion formation after reproductive pelvic surgery. Fertil Steril. 1987, 47:864-866.

31. Marana R, Luciano AA, Marendino VE, et al: Reproductive outcome after ovarian surgery: microsurgery versus CO 2 laser. J Gynecol Surg. 1991, 7:159-162.

32. Lundorff $P$, Hahlin M, Kallfelt B, Thorburn J, Lindblom B: Adhesion formation after laparoscopic surgery in tubal pregnancy: a randomized trial versus laparotomy. Fertil Steril. 1991, 55:911-915.

33. Trimbos-Kemper TC, Trimbos JB, van Hall EV: Adhesion formation after tubal surgery: results of the eighth--day laparoscopy in 188 patients. Fertil Steril. 1985, 43:395-400.

34. Nezhat CR, Nezhat FR, Metzger DA, Luciano AA: Adhesion reformation after reproductive surgery by videolaseroscopy. Fertil Steril. 1990, 53:1008-1011.

35. Operative Laparoscopy Study Group: Postoperative adhesion development after operative laparoscopy evaluation at early second look procedures. Fertil Steril. 1991, 55:700.

36. Polymeneas G, Theodosopoulos T, Stamatiadis A, Kourias E: A comparative study of postoperative adhesion formation after laparoscopic vs open cholecystectomy. Surg Endosc. 2001, 15:41-43.

37. Nascimento MC, Kelley A, Martitsch C, Weidner I, Obermair A: Postoperative analgesic requirements -- total laparoscopic hysterectomy versus vaginal hysterectomy. Aust N Z J Obstet Gynaecol. 2005, 45:140-143.

38. Spaczynski RZ, Duleba AJ: Duleba AJ. Diagnosis of endometriosis. Semin Reprod Med. 2003, 21:193-208.

39. Nezhat F, Nezhat C, Allan CJ, Metzger DA, Sears DL: Clinical and histologic classification of endometriomas. Implications for a mechanism of pathogenesis. J Reprod Med. 1992, 37:771-776.

40. Nezhat C, Nezhat F, Seidman DS: Classification of endometriosis. Improving the classification of endometriotic ovarian cysts. Hum Reprod. 1994, 9:2212-2213.

41. Donnez J, Squifflet J, Jadoul P, Lousse JC, Dolmans MM, Donnez O: Fertility preservation in women with ovarian endometriosis. Front Biosci (Elite Ed). 2012, 4:1654-1662.

42. Nezhat C, Lewis M, Kotikela S, Veeraswamy A, Saadat L, Hajhosseini B: Robotic versus standard laparoscopy for the treatment of endometriosis. Fertil Steril. 2010, 94:2758-2760. 10.1016/j.fertnstert.2010.04.031

43. Seracchioli R, Mabrouk M, Manuzzi L, et al: Post--operative use of oral contraceptive pills for prevention of anatomical relapse or symptom--recurrence after conservative surgery for endometriosis. Hum Reprod. 2009, 24:2729-2735. 10.1093/humrep/dep259

44. Nothnick WB: The emerging use of aromatase inhibitors for endometriosis treatment . Reprod Biol Endocrinol. 2011, 9:87. 10.1186/1477-7827-9-87

45. Soysal S, Soysal ME, Ozer S, Gul N, Gezgin T: The effects of post--surgical administration of goserelin plus anastrozole compared to goserelin alone in patients with severe endometriosis: a prospective randomized trial. Hum Reprod. 2004, 19:160-167.

46. Ferrero S, Camerini G, Seracchioli R, Ragni N, Venturini PL, Remorgida V: Letrozole combined with norethisterone acetate compared with norethisterone acetate alone in the treatment of pain symptoms caused by endometriosis. Hum Reprod. 2009, 24:3033-3041. 10.1093/humrep/dep302

47. Stanley KE Jr., Utz DC, Dockerty MB: Clinically Significant Endometriosis of the Urinary Tract . Surg Gynecol Obstet. 1965, 120:491-498.

48. Sampson JA: Intestinal adenomas of endometrial type . Arch Surg. 1922, 5:217-280.

49. Jenkinson EL, Brown WH: Endometriosis: a study of 117 cases with special reference to constricting lesions of the rectumand sigmoid colon. JAMA. 1943, 122:349.

50. Samper ER, Slagle GW, Hand AM: Colonic endometriosis: its clinical spectrum. South Med J. 1984, 77:912914.

51. Ponka JL, Brush BE, Hodgkinson CP: Colorectal endometriosis. Dis Colon Rectum. 1973, 16:490-499.

52. Berker B, Lashay N, Davarpanah R, Marziali M, Nezhat CH, Nezhat C: Laparoscopic appendectomy in patients with endometriosis. J Minim Invasive Gynecol. 2005, 122:206-209.

53. Nezhat C, Seidman DS, Nezhat F: Laparoscopic surgical management of diaphragmatic endometriosis . Fertil Steril. 1998, 69:1048-1055.

54. Nezhat C, Kazerooni T, Berker B, Lashay N, Fernandez S, Marziali M: Laparoscopic management of hepatic endometriosis: report of two cases and review of the literature. J Minim Invasive Gynecol. 2005, 12:196-200.

55. Joseph J, Sahn SA: Thoracic endometriosis syndrome: new observations from an analysis of 110 cases . Am J Med. 1996, 100:164-170. 